AperTO - Archivio Istituzionale Open Access dell'Università di Torino

\title{
Kriging modelization in predicting metal sheet elongation
}

\section{This is the author's manuscript}

Original Citation:

Availability:

This version is available http://hdl.handle.net/2318/1758365

since 2020-10-14T16:28:41Z

Published version:

DOI:10.1002/qre.2347

Terms of use:

Open Access

Anyone can freely access the full text of works made available as "Open Access". Works made available under a Creative Commons license can be used according to the terms and conditions of said license. Use of all other works requires consent of the right holder (author or publisher) if not exempted from copyright protection by the applicable law. 


\title{
KRIGING MODELIZATION IN PREDICTING METAL SHEET ELONGATION
}

\author{
Grazia Vicario $^{(1)}$, Maria Teresa Giraudo ${ }^{(2)}$ and Valentina Calì ${ }^{(3)}$ \\ (1) Department of Mathematical Sciences, Politecnico di Torino, Torino, Italy \\ (2) Mathematical Department, Università di Torino, Torino, Italy \\ (3) Finsoft I.S. srl, Torino, Italy
}

\begin{abstract}
Modern industries are increasingly replacing real experiments with non-stochastic simulation models due to their restrained costs and growing reliability. The non-stochastic simulator used in this paper is the Finite Element Simulation code (FEM), a widely used numerical technique for the engineering problems modelled by a system of partial differential equations defined on a time-space domain. In such a context, it is common practice to provide a metamodel, a global approximation of the FEM experiment response on the design space to capture local minima/maxima.

We use the most popular metamodel, the Kriging model, applied to an industrial instance: prediction of Bend Deduction. Metal sheet bending is a manufacturing process in which there is a plastic deformation of the work pieces over an axis. This is a metal forming process, similar to other processes where bending changes the shape of the work pieces.

The work focuses on the construction of an optimal initial design in order to achieve a good accuracy of the metamodel at an acceptable computational cost, the theoretical study of this model and the understanding of how it could be conformed to the bend deduction prediction.

The correlation structure, mandatory in a Kriging model, was evaluated by means of the variogram, allowing to refine the correlation specification naturally improving the Kriging predictions. The empirical variograms for each input variable brought to light unusual behaviors. This suggested that the bending angle could be related to the bend deduction according to two different models. It is clear that there is a discontinuity in the relationship between the models, but its exact location is not known. The accuracy achieved was then evaluated using different indicators of robustness and the uncertainty of the leave one out methods.
\end{abstract}

Keywords: LH Designs, Kriging, Variogram, FEM, Bend Deduction, Leave One Out.

\section{Introduction}

In this paper, we address the estimation of the bend deduction, a characteristic which occurs during the elongation of a metal workpiece during a bending process. In order to obtain the desired final geometry of the workpiece, it is essential to predict it as precisely as possible because a high noise in its measurement may lead to a variety of problems. There are two methods commonly used for this prediction: the experimental and the analytical method. We refer to the procedure as experimental even if the Finite Element Method (FEM), a simulated experiment, was employed.

In this context, the crucial points are the design of experiments to be performed according to the input variables, the choice of a metamodel, i.e. a global approximation of FEM experiment response 
on the design space to capture local minima/maxima requiring a more easy numerical evaluation, the detection of a possible correlation among the input variables and the estimation of model parameters. Finally, a discussion of the goodness of the predictions is essential.

The engineering problem of the bend deduction is described in Section 2. Section 3 presents the basic principles of Kriging modelization, the most popular metamodel, and the uncertainty of Kriging predictions for responses of simulated experiments. Section 4 is composed of three subsections according to the focuses of the case study based on real data from FEM applications. Subsection 4.1 describes the choice of an appropriate design of the experiment because the parsimony of the process forces the number of input variables to be small; in 4.2 the detection of the correlation structure among the input variables is presented, with the careful choice of the correlation function via variogram which does not rely on a-priori beliefs; this practice brought to light a peculiarity in the bending process that could seem in contrast with the variogram definition. The last sub-section is devoted to validate the bend deduction predictions according to the methodologies discussed thus far and comparing the results with the predictions obtained either with all the input variables or with data from physical experiments, in order to achieve an adequate trade-off between accuracy and costs.

A final discussion concludes the paper, highlighting the peculiarities, and benefits and drawbacks of the proposed procedure on the basis of the simulated and physical experiments performed.

\section{The engineering problem}

Metal sheet bending is a manufacturing process in which a plastic deformation of the work piece over an axis occurs. The bending process is used in a huge variety of industries, such as general industries, the household electrical appliances industry, the computers industry, the lighting industry, the aerospace industry, the automotive, the medical industries, and so on. As the shape of the work piece is changed, it involves both tension and compression of the materials, and they are highly influenced by technological properties of metals, particularly in the case of plastic and elastic deformation. The metal behavior during the deformation process is well represented by a nonlinear curve, stress-strain curve (see Figure 1); it depicts such behavior distinguishing the initial linear part characterizing the elastic strain from the nonlinear final part which models the plastic phase.

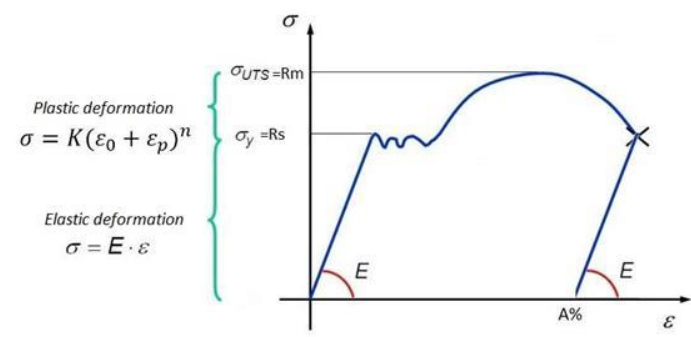

Figure 1: The stress-strain curve depicts the material behaviour during the deformation process. The curve can be decomposed into a linear initial part, characterizing the elastic strain, and a final nonlinear part, that models the plastic phase.

The elastic deformation is compliant with Hooke's law, which asserts that strain is linearly proportional to stress, $\sigma=\mathrm{E} \varepsilon$; the proportionality constant is named Young's Modulus and allows to classify the rigidity of the material. Beyond the level whereby the relationship between stress and strain is no longer linear, a permanent deformation occurs in the material ${ }^{1}$ (Smith, 1994, Chapter 8). 
The stress-strain curve underlines other relevant features of the material: the tensile strength $\left(R_{m}\right)$, that is the maximum load before the sheet breaks, and the yield stress $\left(R_{s}\right)$ that points out the beginning of the plastic deformation of the sheet.

There are three main types of bending 2 : coining, bottoming and air bending. The type used is chosen according to the application and to the function of the final product. We deal with air bending, the most popular in industry. The air bending process consists in positioning a metal work piece that is then pressed into a die by a punch. The punch has to be positioned on the bending line of the workpiece on which the force supplied by the machine power generator is applied (see Figure 2). In turn, the work piece is placed on the die, distinguished by its shape and by the number of grooves. The die affects the internal radius of the work piece, both according to the die width and to the radius of the groove (shoulder radius).

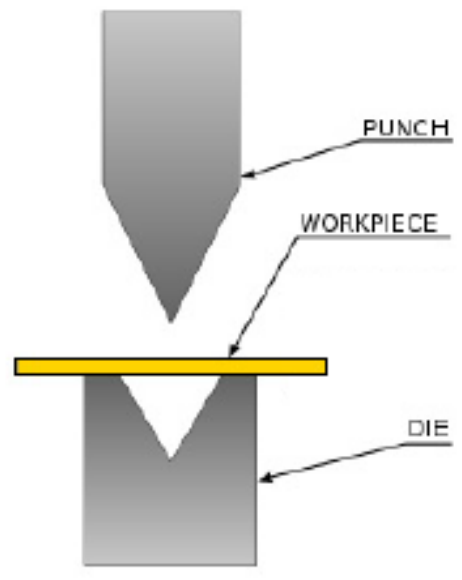

Figure 2: The air bending process: the punch, the die and the workpiece of metal before bending.

This bending process is recognized as a natural event and for this reason it could be considered an easy matter. However, the sheet metal bending is not as simple as it appears. In fact, one of the critical challenges in air bending is to maintain the geometric tolerances of the work piece. If the work piece is a sheet ${ }^{3}$, two phenomena mainly affect its accuracy: the springback problem and the Bend Deduction (plastic elongation or compression). The springback phenomenon consists in the fact that the workpiece springs back a little and the ending angle is more open than desired, i.e. the bent sheet's elastic recovery toward its original shape after bending. Stretching or shrinkage of the metal sheet is the cause of Bend Deduction (BD). In Figure 3 a bent metal sheet is depicted: if a workpiece is bent in two parts with a defined angle $\alpha$ and if F1 and F2 are the flange lengths, the total length of the work piece $\left(\mathrm{L}_{\mathrm{t}}\right)$ will not be F1+ F2. To find out the real new length of the bent work piece it is necessary to compute the $\mathrm{BD}$, i.e. how much to add or subtract to the flange lengths to get exactly the real new length. 


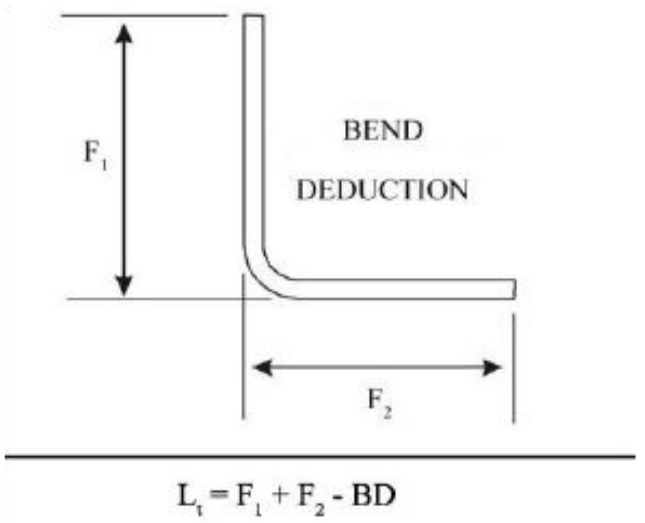

Figure 3: A bent metal sheet is depicted to show the bend deduction and the elongation of the sheet after bending. In the figure, $\mathrm{L}_{t}$, is the total length of the workpiece before the bending, $\mathrm{F}_{1}$ and $\mathrm{F}_{2}$ are the lengths of the flanges and $\mathrm{BD}$ refers to bend deduction.

The aim of the research developed in this paper is to predict BD; the same strategy could be applied for predicting springback. We used the Kriging models with data from simulated experiments with the Finite Element Method. In the next Section, the principle of Kriging is introduced with basics of the modelization of the correlation structure, depending parametrically on the distance between the locations of the observations.

The data analyzed were generated in collaboration with MUSP (Macchine Utensili e Sistemi di Produzione) of Piacenza Tecnopolo (Italy). The name of the company will not be displayed as it is no longer interested in carrying on the research being the obtained accuracy satisfactory.

\section{Essentials of Kriging model}

In principle, when dealing with data from deterministic Computer Experiments (CE), the statistical framework of physical experiments can be applied, allowing that the factors make up a subset of code's inputs. The analogy with the physical experiment is satisfied if each computer run is regarded as a realization of a stochastic process. In fact, in complex codes there is a number of parameters that have no link to the physical problem: implicit or explicit methods for solving differential systems, elementary discretization intervals, convergence thresholds for iterative techniques, and so on. They pertain only to the code and may be deemed as nuisance parameters, in respect of the usual requirements such as non correlation, normal distribution, constant variance ${ }^{4}$.

At the end of the 1980s, a group of statisticians ${ }^{5-6}$ set up a framework using the Kriging model for the deterministic output of computer experiments. These models (distinguished in simple, ordinary and universal), named after the South African engineer Daniel G. Krige ${ }^{7}$, were first introduced for predicting noisy spatial responses from a generally small number of observations. Facing the problem of making accurate predictions of a response basing on a limited set of spatial data, Krige put in statistical terms the rational idea that response values spatially close are much more alike than values that are more distant. Thus, observations closer to untried locations should influence more the prediction at it.

We consider the case with $d$ input variables and one output variable. Let $D \subset \mathbb{R}^{d}$ be the region where we want to predict the response $y$ observed in a set of points (training points).

The notation that will be used is: $\mathbf{x}_{i}=\left(x_{i 1}, \ldots, x_{i d}\right)$, with $\mathbf{x}_{i} \in D$ for $i=1,2, \ldots, n$, is a $1 \times d$ row vector representing the $i$-th training point on the $d$-dimensional input space; $\mathbf{X}$ is the $n \times d$ design matrix 
that can be written as $\mathbf{X}=\left[\mathbf{x}_{1}^{\prime}, \mathbf{x}_{2}^{\prime}, \ldots, \mathbf{x}_{n}^{\prime}\right\rfloor$ and $y_{i}$ is the observed value of the computer code output at the $i$-th input setting. Then, the $n \times 1$ vector of outputs observed at the $n$ design sites is given by $\mathbf{y}^{n} \equiv \mathbf{y}(\mathbf{X})=\left(y\left(\mathbf{x}_{1}\right), y\left(\mathbf{x}_{2}\right), \ldots, y\left(\mathbf{x}_{n}\right)\right)^{\prime}$.

The response $\mathrm{y}(\mathbf{x})$ for $\mathbf{x} \in \mathrm{D}$ from the computer code is modeled ${ }^{5-6}$ as a realization of a Gaussian stochastic process:

$Y(\mathbf{x})=\sum_{j=1}^{k} \beta_{j} f_{j}(\mathbf{x})+Z(\mathbf{x})$,

where $\sum_{j=1}^{k} \beta_{j} f_{j}(\mathbf{x})$ is the usual regression part and $\mathrm{Z}(\mathrm{x})$ is the systematic departure part ${ }^{5} . \mathrm{Z}(\mathbf{x})$ is assumed to be a stochastic process with $\mathrm{E}[\mathrm{Z}(\mathrm{x})]=0, \operatorname{Var}[\mathrm{Z}(\mathrm{x})]=\sigma_{z}^{2}$ and stationary covariance over $D$ so that:

$\operatorname{Cov}[Z(\mathbf{x}), Z(\mathbf{x}+\mathbf{h})]=\sigma_{Z}^{2} R(\mathbf{h} ; \boldsymbol{\theta})$

$R(\mathbf{h}, \boldsymbol{\theta})$ is the stationary correlation function ( $\mathrm{SCF}$ ) depending only on the displacement vector $\mathbf{h}$ between any pair of points in $D$ and on a set of parameters $\theta$. The correlation function needs to be specified, and must satisfy certain conditions, such as positive semi-definiteness, so that we have a valid covariance. The choice of $R(\cdot, \cdot)$ affects the smoothness of the sample paths of the stochastic process $\mathrm{Z}(\cdot)$. We come back to how to estimate the correlation structure in the Sub-section 4.2.

The idea behind the use of the stochastic process is that if we know the response at some $\mathbf{x}_{i}$, we should be able to say something about the response at $\mathbf{x}_{j}, i, j=1, \ldots, n$ and $i \neq j$, depending on how close $\mathbf{x}_{i}$ and $\mathbf{x}_{j}$ are. The Kriging model provides a thorough theoretical framework for predicting. Let $\mathbf{Y}^{n}=\left(Y\left(\mathbf{x}_{1}\right), Y\left(\mathbf{x}_{2}\right), \ldots, Y\left(\mathbf{x}_{n}\right)\right)^{\prime}$ be the set of the output variables at $\mathbf{x}_{i} \in D$ for $i=1,2, \ldots, n$. Then, we assume that, in coherence with (3.1) and (3.2), the joint random variable $\mathbf{Y}^{n+1}=\left(Y\left(\mathbf{x}_{0}\right), Y\left(\mathbf{x}_{1}\right), Y\left(\mathbf{x}_{2}\right), \ldots, Y\left(\mathbf{x}_{n}\right)\right)$ is normal distributed, $\left.\mathbf{Y}^{n+1} \sim N \mid\left(\boldsymbol{f}_{0}^{\prime}, \mathbf{F}\right)^{\prime} \boldsymbol{\beta}, \sigma_{Z}^{2} \Sigma\right\rfloor$, where $\mathbf{f}_{0}$ is the $k$ $\times 1$ vector of regression functions $\mathbf{f}(\mathbf{x})=\left(f_{1}(\mathbf{x}), f_{2}(\mathbf{x}), \ldots, f_{k}(\mathbf{x})\right)^{\prime}$ in $\mathbf{x}_{0} ; \mathbf{F}$ is the $n \times k$ matrix $\left\{f_{j}\left(\mathbf{x}_{i}\right)\right\}_{\substack{i=1, \ldots, n \\ j=1, \ldots, m}}$ of the regression functions computed in $\left(\mathbf{x}_{1}, \mathbf{x}_{2}, \ldots, \mathbf{x}_{n}\right) ; \boldsymbol{\beta}=\left(\beta_{1}, \beta_{2}, \ldots, \beta_{k}\right)^{\prime}$ is the vector of the $k$ regression parameters; $\Sigma=\left(\begin{array}{cc}1 & \mathbf{r}_{0}^{\prime} \\ \mathbf{r}_{\mathbf{0}} & \mathbf{R}\end{array}\right)$ is the covariance matrix with $\mathbf{r}_{0}=\left(R\left(\mathbf{x}_{0}-\mathbf{x}_{1}\right), \ldots, R\left(\mathbf{x}_{0}-\mathbf{x}_{n}\right)\right)^{\prime}$ and $\mathbf{R}=\left\{R\left(\mathbf{x}_{i}-\mathbf{x}_{j}\right)\right\}_{i, j=1, \ldots, n}$ the matrices whose elements are the correlations between the $\mathrm{Z}(\cdot)$ 's at the design sites.

Hence, the mean of $Y(\mathbf{x})$ at the untried point $\mathbf{x}_{0}$ conditional on the process data, $\left.\hat{Y}_{0}=\mathrm{E}\left|Y\left(\mathbf{x}_{\mathbf{0}}\right)\right| \mathbf{Y}^{n}\right]$, is:

$\hat{Y}_{0}=\mathbf{f}_{0}^{\prime} \boldsymbol{\beta}+\mathbf{r}_{\mathbf{0}}^{\prime} \mathbf{R}^{-1}\left(\mathbf{Y}^{n}-\mathbf{F} \boldsymbol{\beta}\right)$

The (3.3) model corresponds to the ordinary Kriging, that reduces the regressive term to a constant $\mathbf{f}_{0}^{\prime} \boldsymbol{\beta}=\beta$, without suffering losses in prediction accuracy ${ }^{5-6}$. Therefore, we use here ordinary Kriging 
because we assume that no a-priori knowledge on $\mathrm{BD}$ is available to drive the choice of $\beta$. This consideration leads consequently to a lower number of parameters to be estimated.

The predictor (3.3) is unique and it is the Best Linear Unbiased Predictor (BLUP) of $Y\left(\mathbf{x}_{0}\right)$, because it minimizes the Mean Squared Prediction Error (MSPE):

$$
\operatorname{MSPE}\left[\hat{Y}_{0}\right]=\mathrm{E}\left(\left(\hat{Y}_{0}-Y\left(\mathbf{x}_{0}\right)\right)^{2}\right)=\sigma_{z}^{2}\left(1-\mathbf{r}_{0}^{\prime} \mathbf{R}^{-1} \mathbf{r}_{0}\right),
$$

MSPE, usually named Kriging variance, is a measure of the uncertainty of predictions: it is large when $\mathbf{x}_{0}$ is far away from the tried points and small when it is close to them; it vanishes at the tried points, owing to the interpolatory property of Kriging.

However, equations (3.3) and (3.4) hold only if $\beta$ and $R(\mathbf{h} ; \boldsymbol{\theta})$ are known, situation that is just unlikely in practical situations. When $\beta$ is unknown, the generalized least squares estimate $\hat{\beta}=\left(\mathbf{F}^{\prime} \mathbf{R}^{-1} \mathbf{F}\right)^{-1} \mathbf{F}^{\prime} \mathbf{R}^{-1} \mathbf{Y}^{n}$ must replace $\beta$ to provide the new predictor. Moreover, in the most common cases the parameter vector $\theta$ in $R(\mathbf{h} ; \theta)$ is unknown. Then, it can be estimated by maximum likelihood, cross-validation, or the posterior model ${ }^{8}$. In such a case the Kriging variance is larger than (3.4):

$$
\mathrm{E}\left(\left(\hat{Y}_{0}-Y\left(\mathbf{x}_{0}\right)\right)^{2}\right)=\sigma_{z}^{2}\left(1-\mathbf{r}_{0}^{\prime} \mathbf{R}^{-1} \mathbf{r}_{0}+\mathbf{c}_{0}^{\prime}\left(\mathbf{F}^{\prime} \mathbf{R}^{-1} \mathbf{F}\right)^{-1} \mathbf{c}_{0}\right)
$$

with $\mathbf{c}_{0}=\mathbf{f}_{0}-\mathbf{F}^{\prime} \mathbf{R}^{-1} \mathbf{r}_{0}$; then, plugging in it the estimates $\hat{\mathbf{r}}_{0}=\mathbf{r}_{0}\left(\hat{\boldsymbol{\theta}}_{\mathrm{ML}}\right)$ and $\hat{\mathbf{R}}=\mathbf{R}\left(\hat{\boldsymbol{\theta}}_{\mathrm{ML}}\right)$, the predictor $\hat{Y}_{0}$ is named Empirical Best Linear Unbiased Predictor (EBLUP).

A notable consequence of using the EBLUP is that the prediction variance is underestimated as it does not account for the extra variability transmitted to $\hat{\mathbf{r}}_{0}, \hat{\mathbf{R}}$ and $\hat{\beta}$ by $\hat{\boldsymbol{\theta}}$. Possible ways to overcome this problem are to resort to an empirical estimate of the variance through parametric bootstrap $^{8}$, cross validation and jackknife ${ }^{9}$.

Given the interpolatory nature of the Kriging predictor, the variance in the tried points is zero, quite an unsuitable situation for modeling noisy data. In order to remedy this, geostatisticians suggest modifying model (3.1) by adding a random error $\varepsilon$ normal distributed with zero mean and constant variance $\sigma_{\varepsilon}^{2}$. The consequence of the model modification does reflect on SCF with the so-called nugget effect ${ }^{10}$.

In this paper, the Kriging modelization was favored in order to estimate BD (see Sub-section 4.2). There are different reasons for this choice: Kriging models work for highly non-linear problems even with an high number of variables; correlation functions make it possible to analyze the effect of every single variable on the response and the user can estimate a confidence interval (the uncertainty of the prediction is provided). Then, the predictions in new points are computed either estimating the parameters of the SCF if the correlation structure is known or estimating the semivariogram through experimental estimators with the aim of finding a parametric model of the correlation. The latter approach was proposed by the pioneers of the Kriging models in geostatistics for predicting noisy spatial responses from a generally small number of observations, a context somewhat different from CE. The semivariogram is defined as: 
$\gamma\left(\mathbf{x}_{i}-\mathbf{x}_{j}\right)=\frac{1}{2} \operatorname{Var}\left[Z\left(\mathbf{x}_{i}\right)-Z\left(\mathbf{x}_{j}\right)\right] \quad$ for all $\mathbf{x}_{i}, \mathbf{x}_{j} \in D$ for $i, j=1,2, \ldots, n$

If the semivariogram depends only on the length of the vector $\mathbf{h}$ between any pair of points $\mathbf{x}_{i}$ and $\mathbf{x}_{j}$, then the stochastic process underlying the semivariogram is isotropic; otherwise, the process is anisotropic. Isotropic processes form an inadequate basis in modelling many spatially distributed data, especially when the monitored manufactured part has technological signatures ${ }^{11}$. In this paper, we favour the use of the variogram for choosing an adequate correlation function, because it is very informative about the random process $\mathrm{Z}(\mathbf{x})$. This differs from the practitioners of the CEs who prefer to use the parametric method, based on either the covariance function or on the correlogram (mostly in time series analysis). The use of variogram is favorite mainly because its estimation, which averages squared differences of the variable, tends to filter the influence of a spatially varying mean (see Section 4). Moreover, the variogram can be applied whenever the first differences of the variable are second-order stationary, a weaker requirement with respect to second-order stationarity. This means that the variogram can be defined also in those cases where the covariance function cannot be defined.

But before dealing with the estimation of the covariance structure, the choice of the training points is mandatory. It is common to use space-filling designs given that the covariance depends parametrically on the distance between the locations. In the next Section, a particular combination of Latin Hypercubes is suggested in order to lower the number of the input variables while preserving their informative contents; moreover, we show how semivariograms can be very effective in detecting a correlation structure, highlighting peculiarities in the data set.

\section{Case study: design, correlation structure and validation of the predictions}

\subsection{Input variables selection and infill designing}

In physical experimentation, the researcher is asked to satisfy a suitable protocol for achieving correct inferences, the Design of Experiments. If a metamodel is used in CEs, a careful selection of the design is fundamental in order to study how the observed response varies when changing the set of input variables. Moreover, when the output of a CE is predicted by assuming Gaussian responses with covariance depending parametrically on the distance between the locations, it is common to use space-filling designs. The basic idea of space-filling designs is a good strategy for the underlying principle of the Kriging model (the nearer an untried point is to the tried points the better is the prediction).

This research development is based on the use of CEs, mainly Finite Elements Method (FEM) simulation with the aim of considering all the variables that might influence sheet behavior in the bending process. A set of twelve variables was firstly considered, specifically:

- material;

- material thickness $\left(t_{0}\right)$;

- Young's module $(E)$ : it measures the resistance of material to elastic deformation, defined according to the relation between the stress and the deformation and depending on the material; 
- percent elongation $(A \%)$ of the material after bending;

- tensile strength $\left(R_{m}\right)$ : it is the maximum load before the sheet breaks;

- yield stress $\left(R_{s}\right)$ : it states the beginning of the plastic deformation of the sheet;

- anisotropy coefficients $r_{x}, r_{x y}$ and $r_{y}$ : the plastic anisotropy of rolled sheet metal is the property according to which the characteristics of the material depend on the direction in which it is considered; in this case this depends on the direction of the lamination of the metal sheet;

- Punch tip radius $\left(R_{p}\right)$;

- Die V-width (w);

- Die shoulder radius $\left(R_{d}\right)$;

- Bend angle $(\alpha)$;

- Friction coefficient $(f)$.

Clearly, the number of input variables is too large. To reduce the number of input variables in the initial design, we adopted statistical methodologies for pinpointing possible relationships between these variables (correlation analysis) and for ranking their importance (General Linear Models). For some specific features, we relied on technological knowledge of the material behavior during the deformation process. The stress-strain curve was used for this analysis; it introduces a constraint in designing the experiment since the yield stress $\left(R_{s}\right)$ has to be smaller than the tensile strength $\left(R_{m}\right)$. Moreover, the literature ${ }^{12}$ suggests summarizing the three anisotropy coefficients $r_{x}, r_{x y}$ and $r_{y}$ in their weighted mean $r_{\text {ave }}$ :

$$
r_{\text {ave }}=\left(r_{x}+2 r_{x y}+r_{y}\right) / 4
$$

Thus, we employed results obtained by previous FEM simulations to confirm our findings. Since the FEM considers the friction coefficient as constant during the simulations, and the two variables punch die radius $\left(R_{p}\right)$ and die shoulder radius $\left(R_{d}\right)$ are only significant for the geometry of the FEM simulations, these variables have been not taken into account in the initial design.

In line with the aforementioned reasoning, the final number of input variables selected was four. These were the material, the die V-width, the bend angle and a variable mixture introduced for taking into account the entangled relationship of the material characteristics: thickness, Young's module, percent elongation and anisotropy coefficients. 
LHS 1

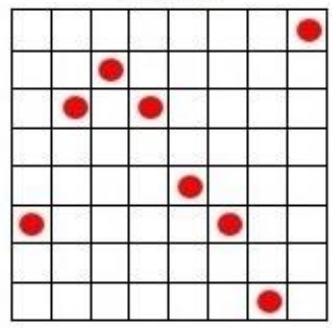

LHS 2

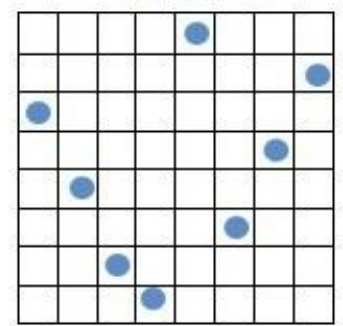

TOTAL DESIGN

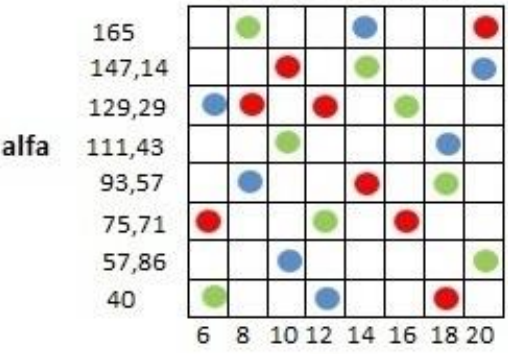

w
LHS 3

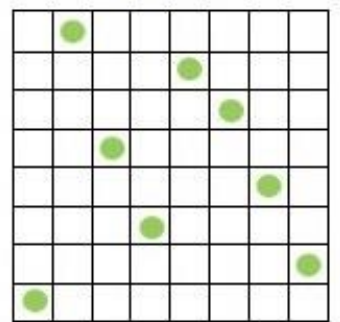

\section{Assembled design \\ with 24 design points}

Figure 4: The three different Latin Hypercubes (LHs) created for the variables die V-width and bend angle, choosing randomly eight sample points in an $8 \times 8$ grid. The last LH is obtained assembling the three previous LHs: the resulting design can be considered satisfactory with respect to the space-filling properties.

A Latin Hypercube ${ }^{13}(\mathrm{LH})$ has been created for the variables die V-width and bend angle, randomly choosing eight sample points in an $8 \times 8$ grid. This LH sampling was repeated three times, considering three different values of the mixture variable. None of the three LH designs created so far may be completely satisfactory if used alone. Hence, it is common practice to use designs constructed by combining several designs: an overall design with 24 sample points obtained assembling the three LHs was created (see Figure 4). The resulting design was considered satisfactory with respect to the space-filling properties. Finally, this procedure was repeated for the four different materials considered in the experiment. The design thus obtained seemed reasonable both for the sample size and for the space-filling requirements.

\subsection{Correlation structure and variogram}

As anticipated in the previous section, empirical semivariograms for each input variable have been estimated in order to model the covariance structure.

A natural estimator of the variogram, the most used in the literature, is based on the method of moments ${ }^{14}$, under the assumption that $\mathrm{E}[\mathrm{Z}(\mathbf{x})]=0$ :

$$
2 C(\mathbf{h})=\frac{1}{\# N(\mathbf{h})} \sum_{N(\mathbf{h})}\left(Z\left(\mathbf{x}_{i}\right)-Z\left(\mathbf{x}_{j}\right)\right)^{2}
$$

where $N(\mathbf{h})=\left\{\left(\mathbf{x}_{i}, \mathbf{x}_{j}\right): \mathbf{x}_{i}-\mathbf{x}_{j}=\mathbf{h} ; i, j=1,2, \ldots n\right\}$ and $\# \mathrm{~N}(\mathbf{h})$ is the number of pairs $\mathrm{N}(\mathbf{h})$ that are distinct. The Matheron estimator (4.1) is unbiased if the Gaussian process is stationary; unfortunately, it may fail in robustness, because it is very sensitive to the presence of outliers (mainly owing to the quadratic term in its definition). To prevent this drawback, other estimators have been proposed in the literature: see Cressie and Hawkins (1980) ${ }^{15}$, Haslett (1997) ${ }^{16}$ and Genton $(1998)^{17}$. Alternatively, outliers should be detected and removed from the set of 
observations, taking into account both physical knowledge and statistical rules for the detection and the removal of outliers. In the case study we dealt with measurement data not seriously affected by the presence of outliers, mainly due to the simulated experiments.

We estimated a semivariogram, according to (4.1), for the input variables considered in the LH initial design: the die $\mathrm{V}$-width and the bend angle. For this purpose, we employed the function variogram in the package geo $\mathrm{R}^{18}$. The design described in 4.1 provides 96 design points and as many data values. It is only a little less than the minimum number required for attaining a steady semivariogram ${ }^{19}$. Moreover, we assume that the semivariogram is isotropic, that is it depends only on the length of the vector $\mathbf{h}$ and not on its direction; the anisotropy may not be properly detected if the sample size is not sufficiently adequate (larger than 300 data). The semivariogram with respect to the die $\mathrm{V}$-width shows a trend compatible with the features of a semivariogram (monotone ascendant, presence of a nugget and a sill); on the other hand, the semivariogram with respect to the bend angle is not comprehensible (see Figure 5-a) and makes it difficult to interpret it as the value of the statistical index in (3.6). In order to interpret the semivariogram in Figure 5-a, we involved the practitioners of the bending process in the company.

They acknowledged that the bending process has different behaviors depending on the values of the bend angles, distinguishing between angles smaller or greater than $90^{\circ}$; this feature is common to all four materials considered in the simulations. We estimated the two semivariograms, one for each interval of values of the variable (see Figure 5-b).

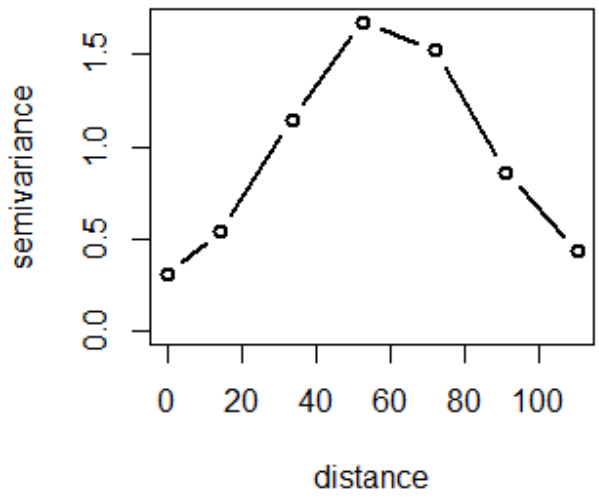

(a)

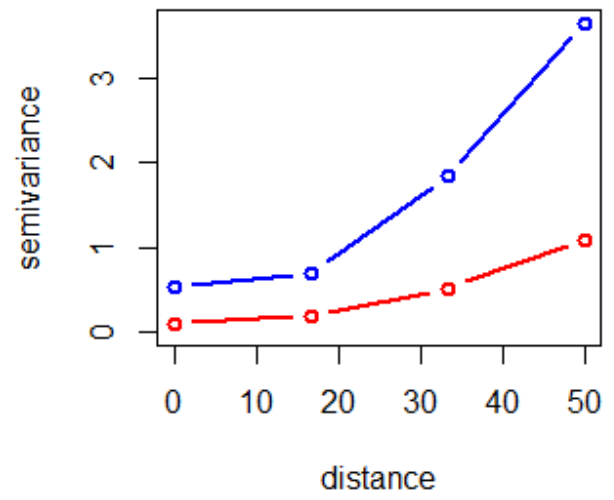

(b)

Figure 5: (a) Empirical variogram with respect to the bend angle; (b) Two empirical variograms with respect to the bend angle considering distinctly the values according to the discontinuity observed in the neighborhood of $90^{\circ}: \alpha \epsilon\left[40^{\circ}, 94^{\circ}\right]$ (blue line) and with respect to $\alpha \in\left[95,165^{\circ}\right]$ (red line). The splitting value was dictated by the differences observed with the four types of materials.

The next step was to fit different models (Matern, exponential, Gaussian and power) suitable for the empirical semivariogram, i.e. the scatter plot of the pair of distances and their corresponding variogram values, making use of the function variofit of the $R$ package geo $R^{18}$. Concerning the three variograms (one for the die V-width and two for the bend angle) the Gaussian model clearly performed better in two situations while in the third it was comparable with the exponential model, with no remarkable differences. The adequacy of the variogram structure in describing the dependencies implicit in the data can be checked a posteriori by computing two statistical indices, with the Leave-one-out (LOO) cross validation procedure ${ }^{19}$ : the Root Mean Squared Error (RMSE) and the squared root of the Mean Square Prediction Error (MSPE). The computation of these 
indices requires the estimate of the Kriging variance and the prediction of the responses in the locations $\mathbf{x}_{i}, i=1, \ldots, n$. Given the Kriging interpolatory feature, we expect that the root MSPE is close to the average standard error and the RMSE is close to one in order for the model to correctly assess the variability in the predictions and estimate the global variability. Any drastic departure from these recommended values is a warning that the model is biased ${ }^{19}$.

It was thus decided to use the Gaussian model for the three covariance structures, obviously with different parameters.

\subsection{Discussion of results}

The sufficient availability of FEM simulated data allowed us to perform a more detailed analysis of the predictions of the $\mathrm{BD}$ with the $\mathrm{R}$ package DiceKriging ${ }^{20}$, considering first the model with the restricted number of input variables, as was discussed in 4.1, and then the model with all the variables. Finally, a validation set with experimental values was used to validate these two models. The DiceKriging provides, in addition to the predictions, graphical tools for analyzing their goodness with the LOO cross validation $\operatorname{method}^{10}$ : the plots of the predicted values against the experimental ones and of the standardized residuals at each of the 96 locations, and the quantilequantile (QQ) plot of residuals.
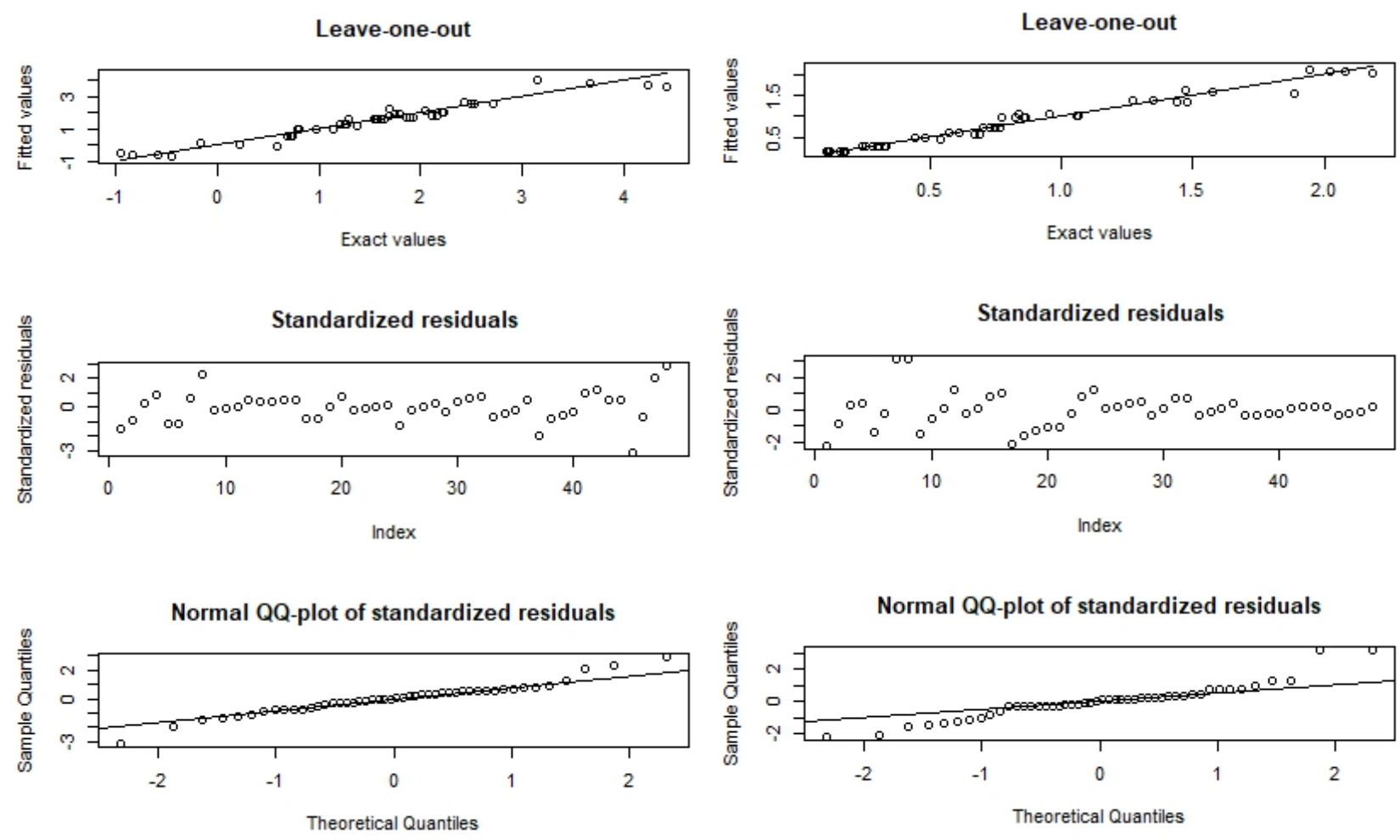

Figure 6: Diagnostic plots for the DiceKriging predictions with the LOO method: in the left panel for $\alpha \in\left[40^{\circ}, 94^{\circ}\right]$ and in the right panel for $\alpha \in\left[95^{\circ}, 165^{\circ}\right]$. At the top there are the scatterplots of exact values versus the predicted ones, in the middle the standardized residuals and at the bottom the normal quantile-quantile (QQ) plots of standardized residuals.

In Figure 6 we plot the two series of diagnostic plots related to the models with the reduced set of variables for $\alpha \in\left[40^{\circ}, 94^{\circ}\right]$ (left) and for $\alpha \in\left[95^{\circ}, 165^{\circ}\right]$ (right). The displays of the LOO analysis 
are fairly good in both cases; the residuals are normally distributed but the larger bend angles present a more visible deviation from Gaussianity.

PERCENTAGE ERROR

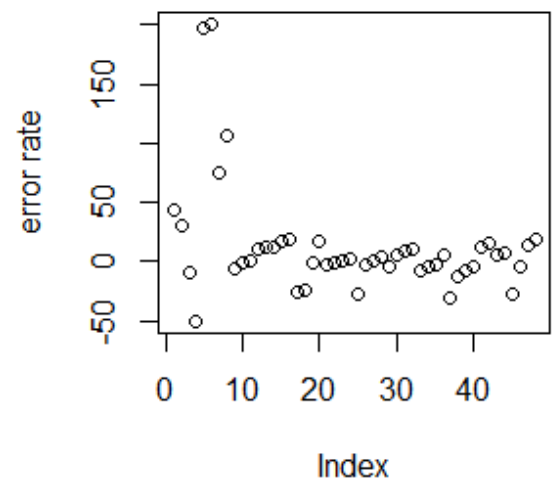

PERCENTAGE ERROR

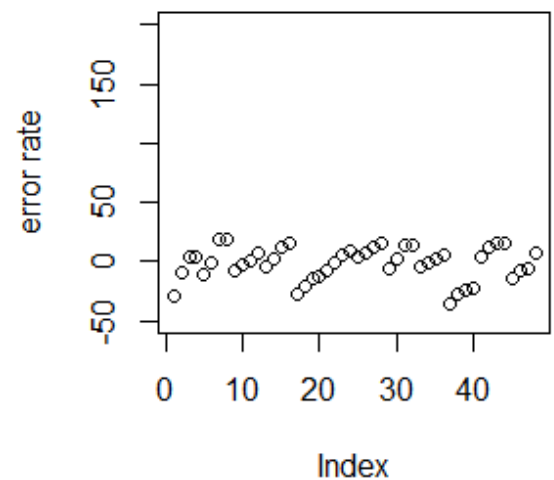

Figure 7: Distribution of the percentage LOO errors for $\alpha \epsilon\left[40^{\circ}, 94^{\circ}\right]$ on the left and for $\alpha \epsilon\left[95^{\circ}, 165^{\circ}\right]$ on the right.

Moreover, we plotted the behavior of the LOO percentage errors for $\alpha \epsilon\left[40^{\circ}, 93.57^{\circ}\right]$ and for $\alpha \epsilon$ $\left[95^{\circ}, 165^{\circ}\right.$ ] (Figure 7). It is evident that the errors in the left panel are not homogeneously distributed; the variability is very large for smaller values of the bend angles. This circumstance was justified by the company's engineers: the bendings are executed with a different technique when the bend angle is small. However, the company is aware that the uncertainty in this case is sizeable. For higher values of bend angles, this problem does not occur, and the percentage errors are uniformly distributed with a constant variability.

As an additional analysis, we considered the Kriging with the full set of input variables. In compliance with the previous model with a reduced set of variables, a Gaussian correlation function was assumed throughout. Also in this case, two different models were employed for the different ranges of the bend angle values.

The values of RMSE and of MSPE (Table 1) were compared with the corresponding average standard errors. The comparison was satisfactory, except in one of the four cases.

\begin{tabular}{|c|c|c|c|c|}
\cline { 2 - 5 } \multicolumn{1}{c|}{} & \multicolumn{2}{|c|}{ Reduced set of input variables } & \multicolumn{2}{c|}{ Full set of input variables } \\
\cline { 2 - 5 } \multicolumn{1}{c|}{} & RMSE & MSPE & RMSE & MSPE \\
\hline$\alpha \in\left[40^{\circ}, 94^{\circ}\right]$ & 0.278 & 1.015 & 0.194 & 0.909 \\
\hline$\alpha \in\left[95^{\circ}, 165^{\circ}\right]$ & 0.107 & 1.005 & 0.309 & 1.030 \\
\hline
\end{tabular}

Table 1: Comparisons of the two statistical indices RMSE and MSPE for different ranges of the bend angles and for full and reduced set of input variables.

Finally, a validation set of real values, made available by the company, was used to validate and to compare the models (reduced and full set of input variables). The mean percentage errors computed for both models are shown in Table 2, according to the different ranges of the bend angles and to the different sets of input variables considered.

\begin{tabular}{|c|c|c|}
\hline & $\begin{array}{c}\text { Reduced set of input variables } \\
\text { Mean percentage error }\end{array}$ & $\begin{array}{c}\text { Full set of input variables } \\
\text { Mean percentage error }\end{array}$ \\
\hline
\end{tabular}




\begin{tabular}{|c|c|c|}
\hline$\alpha \in\left[40^{\circ}, 94^{\circ}\right]$ & $8 \%$ & $3.02 \%$ \\
\hline$\alpha \in\left[95^{\circ}, 165^{\circ}\right]$ & $13.6 \%$ & $5.43 \%$ \\
\hline
\end{tabular}

Table 2: Comparisons of the mean percentage errors considering different ranges of the bend angles and different (reduced and full) sets of input variables.

The mean percentage error is slightly more than doubled when the number of variables in the model is significantly reduced for any bend angle values. The results shown confirm the superiority of the model considering the full set of input variables, which was predictable. The increased amount of error was not considered unsatisfactory by the company, because they take into account the reduction in costs (less variables to be measured). The better performance when smaller bend angles are considered is maintained for both the reduced and the full models.

\section{Conclusions and final remarks}

The case study here considered concerns the metal sheet bending process, a technical procedure widely used in many industries. The issue addressed in this paper is the prediction of bend deduction, which is one of the most crucial problems for engineers because it involves many physical and technological properties of the sheet material. We adopted the ordinary Kriging model because of its power to predict with high accuracy the behaviour of non-linear phenomena. This statistical methodology is supported by the CE literature to approximate the output of deterministic simulations, but the application in the field of the bending process is a novelty. Once again ${ }^{21}$, Kriging models have been used in an industrial context, proving their efficiency in predicting. Even if here the data are derived from repeated FEM simulations, we used variograms for modelling a possible covariance, as geostatisticians do for dealing with very noisy data. A design strategy based on a composition of Latin Hypercube Sampling was used for generating controlled random samples in order to guarantee that all the design points were spread evenly in the input variable region, given that the material properties could not be set randomly for physical reasons.

The analysis of the variograms concerning a specific input variable, the bend angle, brought to light an anomalous behaviour of the correlation in a neighbourhood of $90^{\circ}$, and this feature was comparable for all the four materials considered. This suggested the use of two different covariance models for a more accurate description of how the bend angle could be related to bend deduction. We recommend as a future research development that additional simulations of the bending process with different materials should focus on bend angle in the neighbourhood of $90^{\circ}$ in order to find possible relationships between the discontinuity and different materials.

The design of the experiment and the reduction of the input variables was considered a convincing result by the company. In fact, the accuracy achieved in the predictions has been proved by comparing them with data from a real validation set; the mean percentage error obtained (3\% and $5.4 \%$, depending on the value of the bend angle) was recognized as a satisfactory trade-off between accuracy and costs.

A relevant feature of the application presented in this paper is the possibility of suggesting future developments that could provide more in-depth knowledge of the bending process and consequently improve its quality. Many underlying problems in the process were brought to light during the research work. Not all of these could be further examined, but they suggest the need to consider a broader number of simulations and a larger number of input variables in the simulations (i.e. the variable mixture we adopted to interpret the material characteristics requires dedicated runs of FEM to better understand its behavior and effect). 
To summarize, the Kriging modelization for the predictions and the variograms for detecting the covariance structure can be considered as an encouraging preliminary research step to be used as a guide for further developments.

\section{Bibliography}

1. Smith EH. Mechanical Engineer's Reference Book (12 ${ }^{\text {th }}$ edition). Butterworth-Heinemann: Oxford, United Kingdom, 1994.

2. Diegel O. Bend Works, The fine-art of Sheet Metal Bending. Complete Design Services, 2002.

3. Kim H, Nargundkar N, Altan T. Prediction of Bend Allowance and Springback in Air Bending, Journal of manufacturing science and Engineering 2006; 129(2): 342-351.

4. Romano D, Vicario G. Reliable Estimation in Computer Experiments on Finite-Element Codes, Quality Engineering 2001-02; 14(2): 195-204.

5. Sacks J, Schiller SB, Welch WJ. Designs for Computer Experiments, Technometrics 1989a; 31: 41-47.

6. Sacks J., Welch WJ, Mitchell TJ, Wynn HP. Design and Analysis of Computer Experiments. Statistical Science 1989b; 4: 409-435.

7. Krige DG. A statistical approach to some mine valuations and allied problems at the Witwatersrand. Master's thesis of the University of Witwatersrand, South Africa, 1951.

8. Den Hertog D, Kleijnen JPC, Siem AWD. The Correct Kriging Variance Estimated by Bootstrapping. Journal of the Operational Research Society 2006; 57: 400-409.

9. Kleijnen JPC, van Beers WCM. Application-driven sequential designs for simulation experiments: Kriging metamodeling. Journal of the Operational Research Society 2004; 55 : 876-883.

10. Cressie N. Statistics for spatial data. Wiley: New York, NY, 1993.

11. Ruffa S, Vicario G, Pistone G. Analysis of the Covariance Structure in Manufactured Parts. Communications in Statistics-Theory and Methods 2015; 44(21): 4540-4551.

12. Lankford WT, Snyder SC, Bausher JA. New criteria for predicting the press performance of deep drawing sheets, Transactions of the American Society for Metals 1950; 42: 1197-1225.

13. McKay MD, Beckman RJ, Conover WJ. A Comparison of Three Methods for Selecting Values of Input Variables in the Analysis of Output from a Computer Code, Technometrics 1979; 21(2): 239-245.

14. Matheron G. Traité de géostatistique appliquée. Editions Technip: Paris, France, 1962.

15. Cressie N. Fitting Variogram Models by Weighted Least Squares, Mathematical Geology 1985; 17(5): 563-586.

16. Haslett J. On the ample variogram and the sample autocovariance for non-stationary time series, Statistician 1997; 46: 475-485.

17. Genton MG. Highly robust variogram estimator, Mathematical Geology 1998; 30(2): 213221. 
18. Ribeiro PJ, Diggle PJ. geoR: A package for geostatistical analysis. https://cran.rproject.org/web/packages/geoR/geoR.pdf [2016]

19. Robinson TP, Metternicht G. Testing the performance of spatial interpolation techniques for mapping soil properties, Computers and Electronics in Agriculture 2006; 50: 97-108.

20. Roustant O, Ginsbourger D, Deville Y. R Package DiceKriging. https://cran.rproject.org/web/packages/DiceKriging/DiceKriging.pdf [2015]

21. Vicario G, Craparotta G, Pistone G. Meta-models in Computer Experiments: Kriging versus Artificial Neural Networks, Quality and Reliability Engineering International 2016; 32: 2055-2065. 